\title{
A method to extend flamelet manifolds for prediction of NOx and long time scale species with tabulated chemistry
}

\section{Aymeric Boucher* and Nicolas Bertier}

Onera - The French Aerospace Lab, F-92322, Chatillon, France

E-mail: aymeric.boucher@onera.fr

E-mail: nicolas.bertier@onera.fr

*Corresponding author

\section{Francis Dupoirieux}

Onera - The French Aerospace Lab, F-91123, Palaiseau, France

E-mail: francis.dupoirieux@onera.fr

\begin{abstract}
Numerical simulation is currently used to help design low NOx devices for aircraft engines. In order to improve the prediction of pollutant species, combustion models based on tabulated chemistry have been commonly used in recent years. However, the short time scales of usual flamelet manifolds can lead to errors concerning the NO prediction in post flame area, as shown in this paper. Thus, a new method is proposed to extend the manifold in order to describe the evolution of species with a long characteristic time. This method is tested in the framework of 0D-1D computations. The use of the extended manifold is shown to be necessary to correctly predict the evolution of NO concentration in burnt gases. As an example, the extended and classical manifolds were compared in the framework of 2D simulations of an industrial-like combustor, showing an evident difference on the NO levels predicted in the outlet section.
\end{abstract}

Keywords: tabulated chemistry; combustion modelling; $\mathrm{NO}_{\mathrm{x}}$ formation; pollutant prediction; sustainable aviation.

Reference to this paper should be made as follows: Boucher, A., Bertier, N. and Dupoirieux, F. (2014) 'A method to extend flamelet manifolds for prediction of NOx and long time scale species with tabulated chemistry', Int. J. Sustainable Aviation, Vol. 1, No. 2, pp.181-202.

Biographical notes: Aymeric Boucher is a $\mathrm{PhD}$ student of Ecole Centrale Paris and his work is supported by an ONERA grant.

Nicolas Bertier holds a PhD from Paris VI University and is a Senior Scientist in the Fundamental and Applied Energetics Department of ONERA. 
Francis Dupoirieux holds a PhD from Ecole Centrale Paris and is the Deputy

Head of the Fundamental and Applied Energetics Department of ONERA.

\section{Introduction}

Standards regarding emissions of pollutants by aircraft engines, especially nitrogen oxides $\left(\mathrm{NO}_{\mathrm{x}}\right)$, are more and more stringent. Therefore, tools are required to help understand and predict $\mathrm{NO}_{\mathrm{x}}$ formation in combustion chambers designed with new injection concepts. Numerical simulation is one of these tools. Unfortunately, minor species prediction remains a challenging problem due to the large computational cost induced by detailed chemistry. Tabulated chemistry has been widely used in recent years to overcome this difficulty. Assuming that the combustion in gas turbines occurs in a flamelet mode, where the turbulent flame is considered as a thin laminar flame wrinkled by turbulence, the structure of the thin laminar flame is solved separately, using detailed chemistry, and stored in a look-up table indexed by a few number (usually two) of variables. Then, only the equations of these index variables have to be solved in addition to the Navier-Stokes equations, to describe the mixture properties and composition of the turbulent reactive flow. This reduces greatly the computational cost compared to the direct computation including an equation for each species of a detailed chemistry along with the Navier-Stokes equations. Nevertheless, in the way they are usually generated, chemistry tables only describe the evolution of the mixture on short time scales. To take into account the effects of long residence times necessary for $\mathrm{NO}_{\mathrm{x}}$ modelling, tabulated chemistry needs further modelling. Different methods have been proposed to this aim. Vreman et al. (2008) and Van Oijen and De Goey (2009) added a transport equation for NO, where the reaction rate was taken from the chemistry table. Ihme and Pitsch (2008) and Ketelheun et al. (2011) completed this method by splitting the source term into production and consumption parts, in order to include the local NO concentration in the consumption rate. As will be shown in this paper, those methods still present limitations due to the short time scales involved in the chemistry table used. The computation of an equation for $\mathrm{NO}$ helps account for history in $\mathrm{NO}$ evolution and thus long time scales effects. But, the reaction rates extracted from the table, whatever the strategy of splitting, is still limited by the short time scales of the table, and can not take into account all the long residence time effects. Therefore, we propose a new methodology to generate chemistry tables containing the description of the mixture state for larger time scales. The usual flamelet chemistry table is extended by solving a plug flow reactor (PFR) problem. This makes possible to describe, in the chemistry table, the slow evolution of some species such as $\mathrm{NO}_{\mathrm{x}}$ in burnt gases, with the advantage of having a negligible additional cost to generate the table. The extended table generated with this method is firstly tested in the framework of 0D and 1D premixed flames, before being used in the 2D simulation of a twin annular premixing swirler (TAPS) combustor (or 'multipoint' combustor), which is representative of nowadays aircraft combustion devices, and makes it an interesting target for CFD modelling dedicated to pollutants predictions. 


\section{Tabulated chemistry approach}

Tabulated chemistry is useful to describe the effects of a detailed chemistry with a reduced number of variables. These variables are used to pick up the chemical properties in a manifold generated prior to the simulation. In tabulated chemistry of flamelet type, the main idea is that under certain conditions the flame front is only wrinkled by the turbulence and therefore can be locally described by a laminar flamelet associated to a model accounting for turbulence effects. FPI (Gicquel et al., 2000) and FGM (Van Oijen et al., 2001) are two similar models based on the indexing of a flamelet manifold using two variables, the mixture fraction $Z$, and a normalised progress variable $c$. Commonly used definitions of the progress variable $Y_{c}$ (before normalisation) are either

$$
Y_{c}^{\operatorname{def} 1}=Y_{\mathrm{CO}_{2}}+Y_{\mathrm{CO}}
$$

or

$$
Y_{c}^{\text {def } 2}=\frac{Y_{\mathrm{CO}_{2}}}{M_{\mathrm{CO}_{2}}}+\frac{Y_{\mathrm{H}_{2} \mathrm{O}}}{M_{\mathrm{H}_{2} \mathrm{O}}}+\frac{Y_{\mathrm{H}_{2}}}{M_{\mathrm{H}_{2}}},
$$

where $Y_{X}$ and $M_{X}$ refer respectively to the mass fraction and the molar mass of $X$.

Considering a manifold generated from 1D premixed flames, the mixture fraction enables the FPI/FGM model to select the appropriate flamelet, and the progress variable gives the appropriate position in this flamelet. Indeed the progress variable monotonically evolves from 0 in the fresh gases to a maximum value $Y_{c}^{\max }(Z)$ in the burnt gases. $Y_{c}^{\max }(Z)$ is commonly referred to as $\left.Y_{c}\right|_{e q}$ considering that species included in the definition of $Y_{c}$ have reached the equilibrium. This 'equilibrium' prefix must be considered with care because it does not imply that all species of the mixture have reached the equilibrium as it will be shown in this work. The normalised progress variable is finally obtained following this definition:

$$
c=\frac{Y_{c}}{\left.Y_{c}\right|_{e q}}
$$

Two approaches can be used with a tabulated chemistry model. In a direct approach, all the data (mass fractions, transport properties, temperature,...) are extracted from the table. In an indirect approach, only the chemical reaction rates of the main species are extracted. Obviously, additional data can still be extracted directly from the table if necessary. In the direct approach, only the indexing variables of the flamelet manifold are transported in addition to the Navier-Stokes equation system, while with the indirect approach the main species are transported. In this study, we are using the second approach because it offers more flexibility regarding its implementation in the Cedre code. Thus, laminar flamelet reaction rates are stored in a chemistry table. In order to prevent deviation of local composition from the table due to three-dimensional diffusive and convective effects, a correction term is introduced following the previous work 
of Savre et al. (2008) and the reaction rates of the chemical species are generally introduced in the transport equations in the following form:

$$
\begin{aligned}
& \dot{\omega}_{i}(x, y, z, t)=\dot{\omega}_{i}^{T A B}[Z(x, y, z, t), c(x, y, z, t)] \\
& +\frac{Y_{i}^{T A B}[Z(x, y, z, t), c(x, y, z, t)]-Y_{i}(x, y, z, t)}{\tau}
\end{aligned}
$$

The difference with the work of Savre et al. (2008) is that a single characteristic time $\tau$ is used since deviations from table composition are driven by three-dimensional diffusive and convective effects common to all species. It can be verified in the calculations that in each cell of the mesh, the local composition is close to the tabulated one. Therefore, apart from small numerical errors, the results obtained with the indirect approach of tabulated chemistry including the relaxation term, are equivalent to the results one would have with the direct approach. Finally, mass conservation is achieved by calculating the nitrogen $\mathrm{N}_{2}$ (the concentration of which is high compared to other species) source term as:

$$
\dot{\omega}_{N_{2}}(x, y, z, t)=-\sum_{i} \dot{\omega}_{i}(x, y, z, t),
$$

where the summation is made over all the other transported species.

The total energy conservation is solved in the Cedre CFD code and does not require particular treatment since the total enthalpy equation is not affected by chemical source terms. Nevertheless, mass compensation of some tabulated species by $\mathrm{N}_{2}$ leads to a change of the mixture properties. In rich mixtures (FAR $>1$ ), it can induce important changes in heat capacity and formation enthalpy. This affects the temperature calculated from the total energy and from these mixture properties. Flows with moderate FAR do not require additional modelling. But for rich mixtures, a method similar to the one proposed by Galpin (2007) can be used to select species for which replacing their mass by same $\mathrm{N}_{2}$ mass has an important impact on mixture properties. Those species have then to be transported. For exemple, Galpin (2007) showed that modelling combustion of the methane requires to transport $\mathrm{H}_{2}$ in addition to the main species $\mathrm{C}_{10} \mathrm{H}_{22}, \mathrm{O}_{2}$, $\mathrm{H}_{2} \mathrm{O}, \mathrm{CO}_{2}, \mathrm{CO}$ and $\mathrm{N}_{2}$. The value of the normalised progress variable $c$ is calculated from the transported species using equations (1) and (3). An additional equation is solved for the mixture fraction $Z$. The chemistry-turbulence interaction can be modelled using different approaches (presumed Probability Density Function, thickened flame model...). For the sake of brevity, this subject is not addressed in this paper. The reader interested in this topic is referred to the papers describing the existing models (PCM-FPI (Fiorina et al., 2005), F-TACLES (Fiorina et al., 2010), TFLES/ATF (Kuenne et al., 2011)). Nevertheless, it is worth noting that the approach developed in this work offers compatibility with all of these turbulence interaction closures.

To deal with two-phase flows when liquid fuel is injected, we add a source term $\dot{\omega}_{Z}^{\text {evap }}$ in the mixture fraction equation as done by Baba and Kurose (2008). This source term takes into account the creation of atomic carbon and hydrogen when fuel evaporates:

$$
\dot{\omega}_{Z}^{e v a p}=\frac{d Z}{d t}=\frac{d\left(Y_{C}+Y_{H}\right)}{d t}
$$


This source term is generally obtained from the evaporation rates of the different components of the fuel (usually reduced to $\mathrm{C}_{10} \mathrm{H}_{22}$ in computations):

$$
\dot{\omega}_{Z}^{\text {evap }}=\mathcal{M}_{C} \sum_{j} \frac{n_{C, j}}{\mathcal{M}_{j}} \dot{\omega}_{j}^{\text {evap }}+\mathcal{M}_{H} \sum_{j} \frac{n_{H, j}}{\mathcal{M}_{j}} \dot{\omega}_{j}^{\text {evap }}
$$

where $\mathcal{M}_{C}$ and $\mathcal{M}_{H}$ are respectively the atomic weights of carbon and hydrogen, $n_{C, j}$ and $n_{H, j}$ are the number of carbon atoms and hydrogen atoms in the $j^{\text {th }}$ component of the fuel, and $\dot{\omega}_{j}^{\text {evap }}$ and $\mathcal{M}_{j}$ are the mass evaporation rate and molecular weight of the $j^{\text {th }}$ component of the fuel.

The additional modelling used in this work concerns the specific treatment of nitric oxide (NO) and is presented in the next section.

\section{$3 \quad \mathrm{NO}_{\mathrm{x}}$ modelling}

\subsection{NO models based on tabulated chemistry}

Tabulated chemistry gives access to minor species concentrations. Pollutant species such as $\mathrm{NO}_{\mathrm{x}}$ are thus a good target for this model. Unfortunately flamelet tabulation gives rise to difficulties for $\mathrm{NO}_{\mathrm{x}}\left(\mathrm{NO}, \mathrm{NO}_{2}\right)$ prediction as indicated thereafter. In the following, we focus on the $\mathrm{NO}$ formation since the amount of $\mathrm{NO}_{2}$ produced is less important than the amount of NO inside the combustion chamber.

The usual progress variable based on main species [such as in equation (1)] is not adapted to discretise correctly the NO mass fraction gradients in the manifold. Indeed, due to different chemical time scales, large variations of NO mass fraction can occur in a very short part of the $Y_{c}$ domain, leading to large interpolation errors. This problem can be addressed by including the NO mass fraction in the definition of the progress variable (Godel et al., 2009), or by transporting NO instead of directly obtaining it from the table (Vreman et al., 2008). Van Oijen and De Goey (2009) have shown that combining these two approaches gives more accurate results.

Another issue with flamelet tabulation is that species evolving with long time scales cannot be accounted for in usual flamelet manifolds without additional treatment. Indeed, when the progress variable $Y_{c}$ reaches its maximum value in the flamelet, the concentration of the main species does not evolve anymore because they are almost at equilibrium, i.e., their chemical rate is close to zero. Consequently, the concentration of minor species such as NO does not evolve anymore as well, although these species did not actually reach the equilibrium. This can lead to a non-physical saturation effect on NO concentration as pointed out in this work. Solving an additional equation for NO can be considered as a first step to take into account the NO evolution outside the manifold. Nevertheless, the NO concentration obtained in the CFD calculation can be strongly different from the value given by the flamelet calculation. This deviation of the NO concentration from the tabulated trajectories is not taken into account with the tabulated source term, leading to wrong results. Some authors (Ihme and Pitsch, 2008; Ketelheun et al., 2011) recommend to split the source term into production and consumption parts. The production term (here referred to as prod) is obtained directly from the table, whereas the consumption term (here referred to as cons) combines 
the tabulated consumption rate $\left(\frac{\dot{\omega}_{N O}^{\text {cons }}}{Y_{N O}}\right)$ and the NO mass fraction given by the CFD calculation. The reaction rate is evaluated as

$$
\begin{aligned}
& \dot{\omega}_{N O}^{\text {prod }}(x, y, z, t)=\left.\dot{\omega}_{N O}^{p r o d}[Z(x, y, z, t), c(x, y, z, t)]\right|_{\mathrm{TAB}} \\
& \dot{\omega}_{N O}^{c o n s}(x, y, z, t)=\frac{\left.\dot{\omega}_{N O}^{c o n s}[Z(x, y, z, t), c(x, y, z, t)]\right|_{\mathrm{TAB}}}{Y_{N O}^{T A B}[Z(x, y, z, t), c(x, y, z, t)]} Y_{N O}(x, y, z, t) .
\end{aligned}
$$

In this approach, the dependence of the consumption rate on $Y_{N O}$ makes that it is not useful to introduce the relaxation term of equation (4) in the expression of the NO source term. However, it must be kept in mind that if the local composition is accounted for in the NO consumption rate through the NO mass fraction, this is not the case for the NO production rate. Indeed, the latter is still obtained assuming a composition in the CFD calculation close to the composition given by the table. The slow evolution of the other reactants of the $\mathrm{NO}$ mechanism (such as $\mathrm{NO}_{2}$ ) is not taken into account. In the next sections, it will be shown that this approach, with usual flamelet manifolds, leads to the saturation of the NO concentration, leading to an important underestimation of the NO concentration when the residence time is large.

\subsection{Extension of a flamelet manifold by reactor calculations}

In order to overcome the difficulties encountered by the existing methods, i.e., in order that source terms correctly reproduce the slow evolution of NO, a method consisting in the extension of the flamelet manifold is proposed. This method is presented here for a premixed flamelet manifold, but could be adapted to non-premixed ones with moderate effort. Figure 1 shows the evolution of main species and NO mass fractions in a laminar premixed flame calculated by the Cantera software (Goodwin et al., 2013), for a fuel-air equivalence ratio equal to 0.7 . The $\mathrm{C}_{10} \mathrm{H}_{22}$ /air chemistry is modelled using the mechanism of Luche (2003) that contains 91 species. The NO chemistry (prompt and thermal) is included in the mechanism. $\mathrm{C}_{10} \mathrm{H}_{22}$ that is usually used to model aeronautical fuels in such calculations is assumed to be pre-vapourised since the manifold only contains gaseous flames as already explained.

On the lower part of the figure a zoom displays the domain used to generate the usual flamelet manifold (the 'Initial flamelet' part). The main species have reached a state really close to equilibrium at the end of this domain. Therefore, after an early increase, the progress variable based on these main species is nearly constant. On the upper part of the figure, we have the evolution obtained using Cantera when the length of the computational grid is increased in order to capture the full evolution of NO. The progress variable based on main species $\left(Y_{c}^{\text {def1 }}=Y_{C O}+Y_{C O_{2}}\right)$ is constant while the NO concentration still evolves. It is obvious that an important part of the NO evolution cannot be described without the extended part of the domain. Thus, we recommend to include the full length of the extended domain in the flamelet manifold in order to describe correctly the slow processes occurring in the NO formation (and the definition of the progress variable $Y_{c}$ also has to be adapted as discussed hereafter).

Nevertheless, computing a flamelet on a large domain can be difficult in practice, since the size of the domain needed to capture the full evolution of long time species is not known in advance, and can require repetitive domain enlargements and calculations, making the process CPU time consuming. Thus, we propose an alternative method to 
generate the extension of the flamelet. Considering that in the extended part (right) of the domain, composed of burned gases, the gradients of the species concentrations are close to zero, the diffusive fluxes can be neglected, and the 1D premixed problem is similar to a PFR problem. Therefore, we propose to extend the flamelet manifold with PFR calculations. A PFR is solved for each mixture fraction of the manifold. The input state of the mixture introduced in the PFR is the state of the corresponding flamelet taken at $x_{f l m t}$, the abscissa where the progress variable $Y_{c}^{d e f 1}$ reaches its maximum value, i.e., where the main species are close to equilibrium. In Figure 1 it corresponds to the composition obtained at the abscissa where the line separating 'Initial flamelet' from 'Extended part' is drawn. Then, the PFR calculation is carried out until the variation of all species concentrations, in particular those undergoing a slow evolution such as NO, become negligible.

Figure 1 Visualisation of the extended flamelet for a FAR of 0.7 , with a zoom on the initial flamelet (see online version for colours)

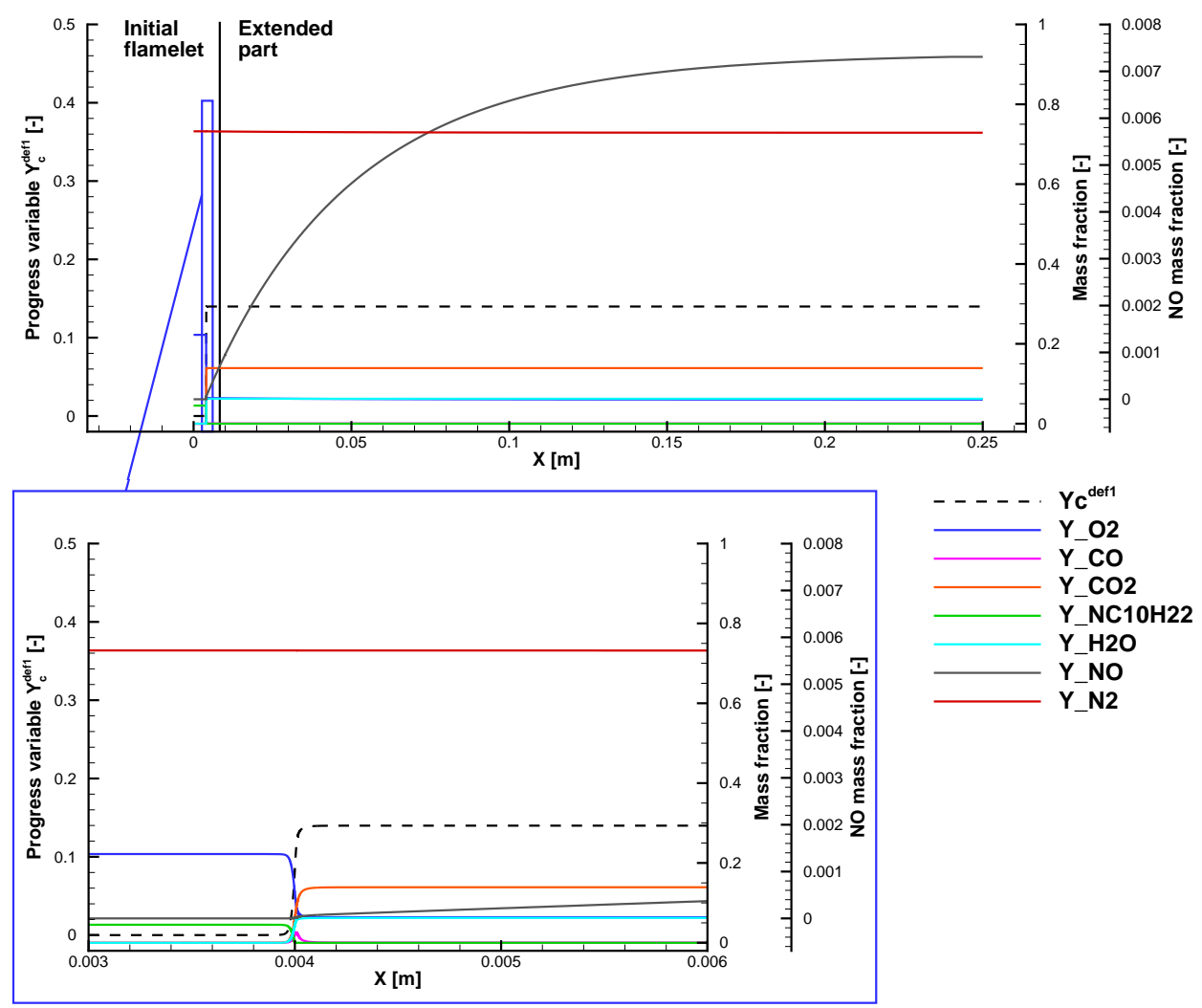

The extension by PFR calculation of a flamelet obtained on a short domain is equivalent to the direct computation of the flamelet on a domain large enough to capture the full evolution of species having long formation time, as shown in Figure 2. 
Figure 2 Comparison of the solution of 1D equations on the enlarged domain (lines) and the solution of a PFR calculation (symbols) (see online version for colours)

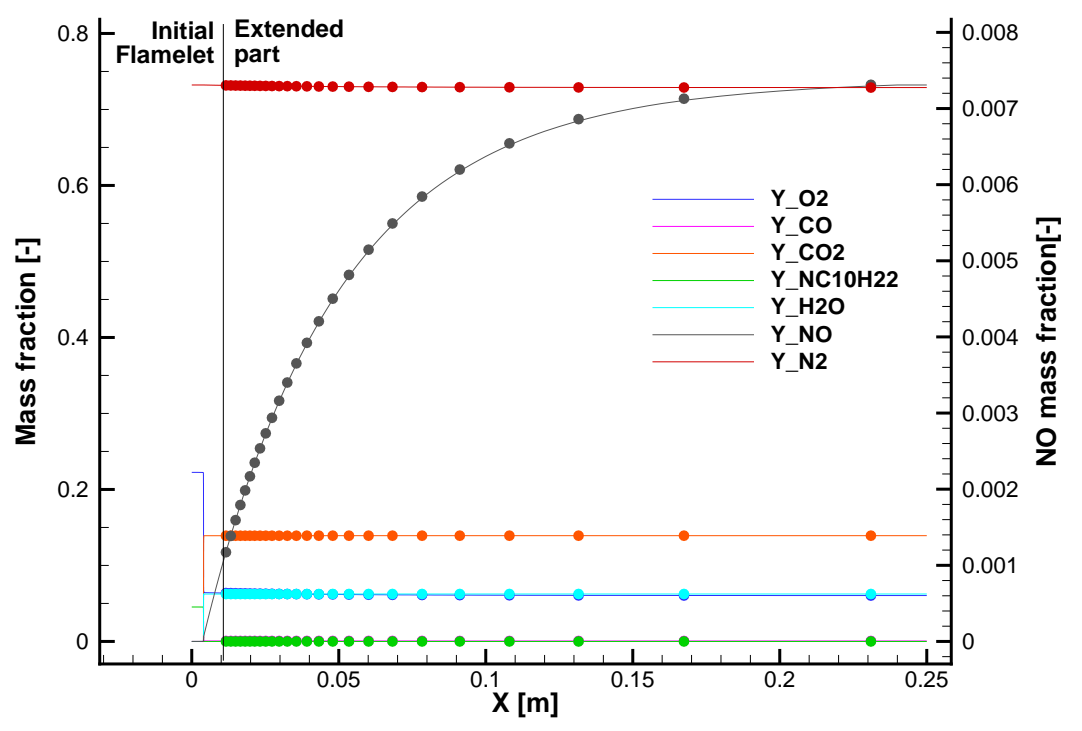

The proposed method based on PFR calculations is easy to implement, and the additional CPU cost induced by the calculation of a PFR is negligible compared to the CPU cost needed to calculate the not extended flamelet.

Once the new extended manifold is obtained, it is used exactly as the not extended flamelet manifold. Only the definition of the progress variable is different in order to remain monotonic as function of $x$ and to take NO into account. Thus the definition proposed by Van Oijen and De Goey (2009) has been considered:

$$
Y_{c}=Y_{c}^{i n i t}+\kappa Y_{N O}
$$

where $Y_{c}^{\text {init }}$ corresponds to the initial definition of the progress variable (such as $Y_{c}^{\text {def } 1}$ or $Y_{c}^{d e f 2}$ ) and $\kappa$ is equal to 10 . Although a value of $10^{3}$ is recommended by Van Oijen and De Goey (2009) for $\kappa$, this value of $\kappa$ is large enough to minimise interpolation errors. The reason is that the maximum value reached by NO is higher with the PFR extension than in the not extended flamelet manifold. Thus the profile of NO is correctly discretised in the manifold. Moreover, the distribution of the grid points of the manifold in the progress variable direction is adapted to take into account the strong variations of the reaction rate. This helps correctly discretise the flamelet, even with small $\kappa$ values. The definition of the progress variable can be modified to include $\mathrm{NO}_{2}$ if necessary, but including NO alone was sufficient to obtain a monotonic evolution of the variable along the flamelet. The $\mathrm{NO}_{2}$ mass fraction can be directly extracted from the table, to access all $\mathrm{NO}_{\mathrm{x}}$ species concentrations. The progress variable used with the extended flamelet manifold is finally:

$$
Y_{c}^{d e f 3}=Y_{C O}+Y_{\mathrm{CO}_{2}}+10 \times Y_{\mathrm{NO}}
$$

It should be noted that the extended flamelet manifold is presented for $\mathrm{NO}_{\mathrm{x}}$ applications, but can be generalised to other species. In particular the method is also attractive for 
CO modelling in rich mixtures, where this species still reacts late downstream the flame front and thus, must be captured by PFR extension of the manifold.

\subsection{NO model comparison for OD time evolving simulation}

In order to compare the behaviour of the different approaches presented for NO formation modelling, some reactor simulations by a CFD code using tabulated chemistry are presented. It is assumed that the reactor contains only burnt gases at the initial time. The composition and temperature of the burnt gases are taken from the flamelet manifold for a given equivalence ratio, at the maximum value of the progress variable $\left(Y_{c}^{\operatorname{def} 1}=\left.Y_{c}^{\operatorname{def} 1}\right|_{e q}\right)$, i.e., the inlet gases are burnt gases in which the main species have reached equilibrium. Then, the reactor is simulated considering constant enthalpy and pressure evolution. In this way, the mixture evolution is representative of the spatial evolution of the burnt gases in a steady state burner such as an aircraft combustor ${ }^{1}$. The chemical mechanism used is the skeletal mechanism for kerosene of Luche (2003). It includes thermal and prompt NO reactions.

Three approaches are compared:

- Model A: An additional transport equation for NO is solved with the source term obtained from the initial flamelet manifold (i.e., not extended) as done by Vreman et al. (2008) and Van Oijen and De Goey (2009). The initial state of the reactor implies that the progress variable has already reached its maximum value. The NO reaction rate is thus extracted from the manifold at the given equivalence ratio, and at the maximum value of the progress variable. This implies that the NO reaction rate is independant of the calculated NO concentration. As in models $\mathrm{B}$ and $\mathrm{D}$ presented hereafter, the relaxation term of equation (4) is not introduced in the NO source term in order that the slow evolution of NO in the post-flame can be reproduced. The use of the relaxation term would have stopped the evolution of $\mathrm{NO}$ at the maximum level given by the not extended manifold.

- Model B: The NO reaction rates are given by equations (8) and (9). Thus, similarly to model $\mathrm{A}$, the NO production rate and the term $\frac{\dot{\omega}_{N O}^{c o n s} \mid \mathrm{TAB}}{Y_{N O T A B}}$ are extracted from the manifold at the given equivalence ratio, and at the maximum value of the progress variable.

- Model C: The NO reaction rate is extracted from the extended manifold. Unlike model $\mathrm{B}$, the NO mass fraction given by the CFD calculation is not involved in the NO consumption term. Instead, it is accounted for in the progress variable definition $\left(Y_{c}^{\operatorname{def} 3}\right)$. In model $\mathrm{C}$, the slow evolution of $\mathrm{NO}$ is taken into account by the extended part of the manifold. Note that the results obtained with model $\mathrm{C}$ are in this particular case (PFR) identical by construction to the results obtained when solving directly the full set of transport equations in the CFD calculation. For that reason the results obtained with model $\mathrm{C}$ are considered as the reference for this simulation.

To sum it up, the three models correspond to the following evaluation of the NO source term in Table 1. 
Table 1 NO source term formulation and type of manifold used in OD calculations

\begin{tabular}{|c|c|c|}
\hline Model & NO source term & Type of manifold \\
\hline A & $\dot{\omega}_{N O}=\dot{\omega}_{N O}(Z, c=1)$ & Not extended \\
\hline B & $\begin{aligned} \dot{\omega}_{N O}= & \dot{\omega}_{N O}^{\text {prod }}\left(Z, Y_{c}=Y_{c}^{\max }\right) \\
& +\frac{Y_{N O}}{Y_{N O}\left(Z, Y_{c}=Y_{c}^{\max }\right)} \dot{\omega}_{N O}^{\text {cons }}\left(Z, Y_{c}=Y_{c}^{\max }\right) \\
= & f\left(Z, c=1, Y_{N O}\right)\end{aligned}$ & Not extended \\
\hline $\mathrm{C}$ & $\dot{\omega}_{N O}=\dot{\omega}_{N O}\left(Y_{i}, T\right)=\dot{\omega}_{N O}(Z, c=c(t))$ & Extended by PFR calculations \\
\hline
\end{tabular}

Figure 3 Comparison of NO models in simulations of a PFR at constant enthalpy and pressure (see online version for colours)
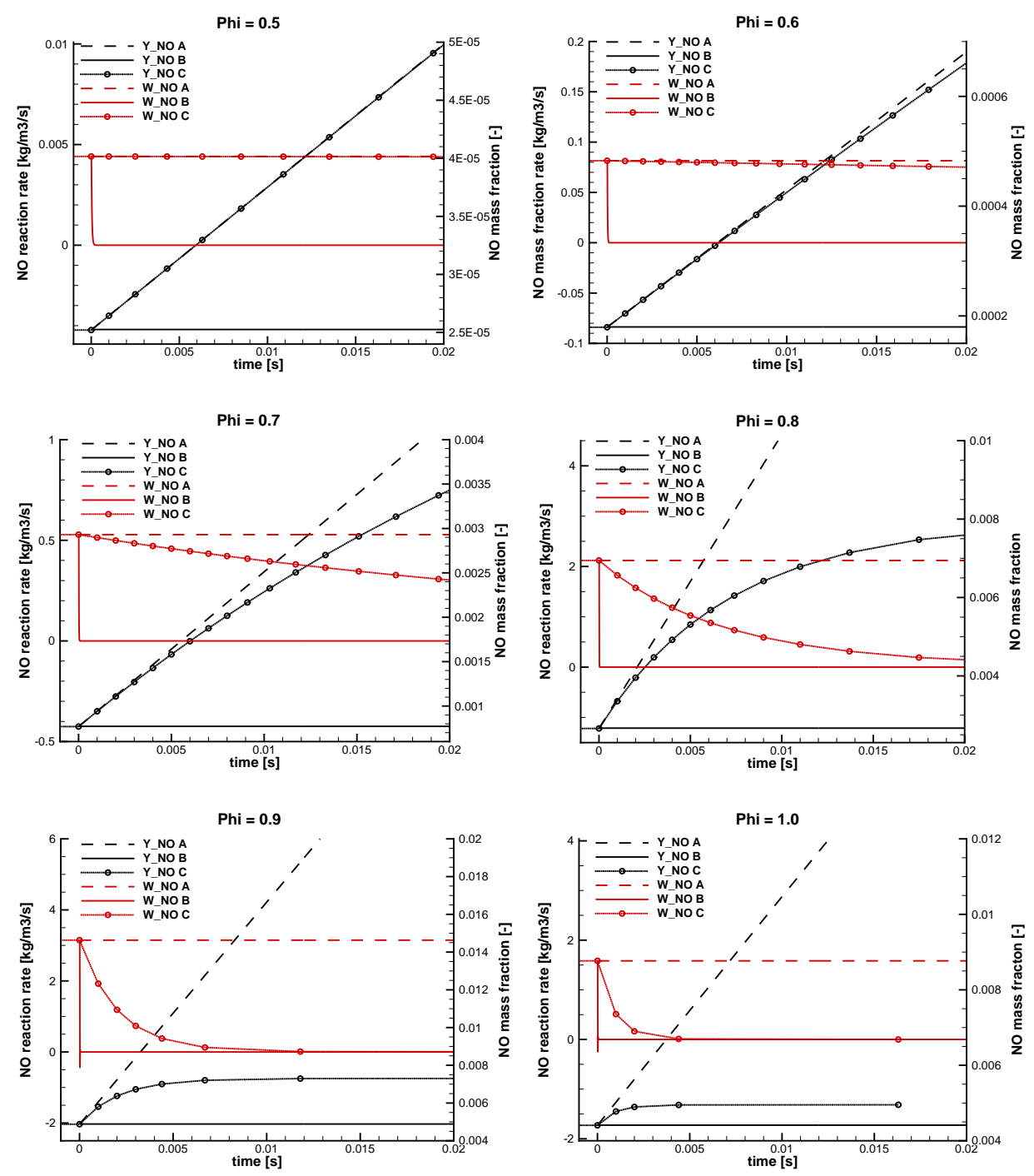
The results obtained with the three approaches are compared in Figure 3. The evolution of the NO mass fraction and NO source term are plotted versus time for each model, for different equivalence ratios. Only the first $20 \mathrm{~ms}$ of the simulation are presented since the residence time in aeronautical engines we are interested in usually lies between $3 \mathrm{~ms}$ and $20 \mathrm{~ms}$.

The relevant information deduced from these results is:

- As mentioned previously, the model A gives a source term independent of the calculated NO mass fraction. It implies a linear evolution of $Y_{N O}$. This behaviour is close to the evolution predicted by model $\mathrm{C}$ for lean mixtures. But for mixture closer to the stoichiometry, the NO concentration is over-predicted. Thus, the accuracy of this model will strongly depend on the equivalence ratio of the burnt gases and can not be considered as predictive.

- Model B predicts a nearly constant value of NO mass fraction. This is explained by the constant value of the production term in the model. A small increase of $Y_{N O}$ makes possible the balance between the consumption rate and the constant production rate. Finally, the value predicted by the model B is very close to the value obtained at $c=1$ in the not extended manifold since the net NO source term is in practice negligible in the PFR. This is what we call the saturation effect, which occurs with usual flamelet manifolds, and it is not possible to solve this problem without further modelling.

- As mentioned earlier, the model $\mathrm{C}$ is by construction equivalent to solving directly the full set of transport equations in the CFD calculation, leading to a correct reproduction of the NO evolution. The extension of the flamelet manifold through PFR calculations appears as an interesting approach in these preliminary simulations. It is further investigated in a one-dimensionnal case in the next section.

\section{NO modelling analysis in the framework of 1D laminar premixed flame}

The results corresponding to the unstretched premixed flame presented in Figures 1 and 2 were obtained by Cantera (Goodwin et al., 2013) using the full chemical mechanism of Luche (2003). To complete the comparison between the different NO modelling approaches, this flame was simulated in Cedre with the three approaches summarised in Table 1. In the previous section, we showed that model B can not overcome what we call the saturation effect of the not extended flamelet manifold. Nevertheless, this approach allows deviations from the tabulated trajectory controlled by the ratio $\frac{Y_{N O}(x, y, z, t)}{Y_{N O}^{T A B}[Z(x, y, z, t), c(x, y, z, t)]}$. These deviations account for the NO history and comply with the fact that NO does not relax instantaneously to the tabulated trajectories (Ihme and Pitsch, 2008). This formulation of the reaction rates is consistent with the slow reaction rates of NO. Therefore, we propose to test a fourth approach (model D) which is the formulation of the NO reaction rate according to equations (8) and (9), but with the extended flamelet manifold used in model C. All four models are tested with the same $Y_{c}^{\text {def3 }}$ definition that includes NO. It must be kept in mind that the 'equilibrium' values $Y_{c}^{d e f 3} \mid e q$ of the progress variable is higher in the extended manifold of models $\mathrm{C}$ and $\mathrm{D}$, than in the not extended manifold of models $\mathrm{A}$ and $\mathrm{B}$. 
In effect, $Y_{c}^{\text {def } 3} \mid e q$ defined as $\max \left(Y_{c}^{\text {def } 3}(Z)\right)$, is higher in the extended manifold due to higher NO concentration. Table 2 summarises the four approaches compared in this section.

As mentioned previously, models A, B and D do not contain the relaxation term of equation (4) in the NO source term. Therefore, they are consistent with the formulation used in the literature.

Table 2 NO source term formulation and type of manifold used in 1D calculations

\begin{tabular}{lcc}
\hline Model & \multicolumn{1}{c}{ NO source term } & Type of manifold \\
\hline A & $\dot{\omega}_{N O}=\dot{\omega}_{N O}(Z, c)$ & Not extended \\
B & $\dot{\omega}_{N O}=\dot{\omega}_{N O}^{\text {prod }}(Z, c)+\frac{Y_{N O}}{Y_{N O}\left(Z, Y_{c}=Y_{c}^{\text {max }}\right)} \dot{\omega}_{N O}^{\text {cons }}(Z, c)$ & not extended \\
C & $\dot{\omega}_{N O}=\dot{\omega}_{N O}(Z, c)$ & extended by PFR calc. \\
D & $\dot{\omega}_{N O}=\dot{\omega}_{N O}^{\text {prod }}(Z, c)+\frac{Y_{N O}}{Y_{N O}\left(Z, Y_{c}=Y_{c}^{\text {max }}\right)} \dot{\omega}_{N O}^{\text {cons }}(Z, c)$ & extended by PFR calc. \\
\hline
\end{tabular}

Figure 4 NO mass fraction in laminar unstretched premixed flame with FAR $=0.7$, calculated with four different approaches to model NO formation, compared to full chemistry result obtained in Cantera (see online version for colours)

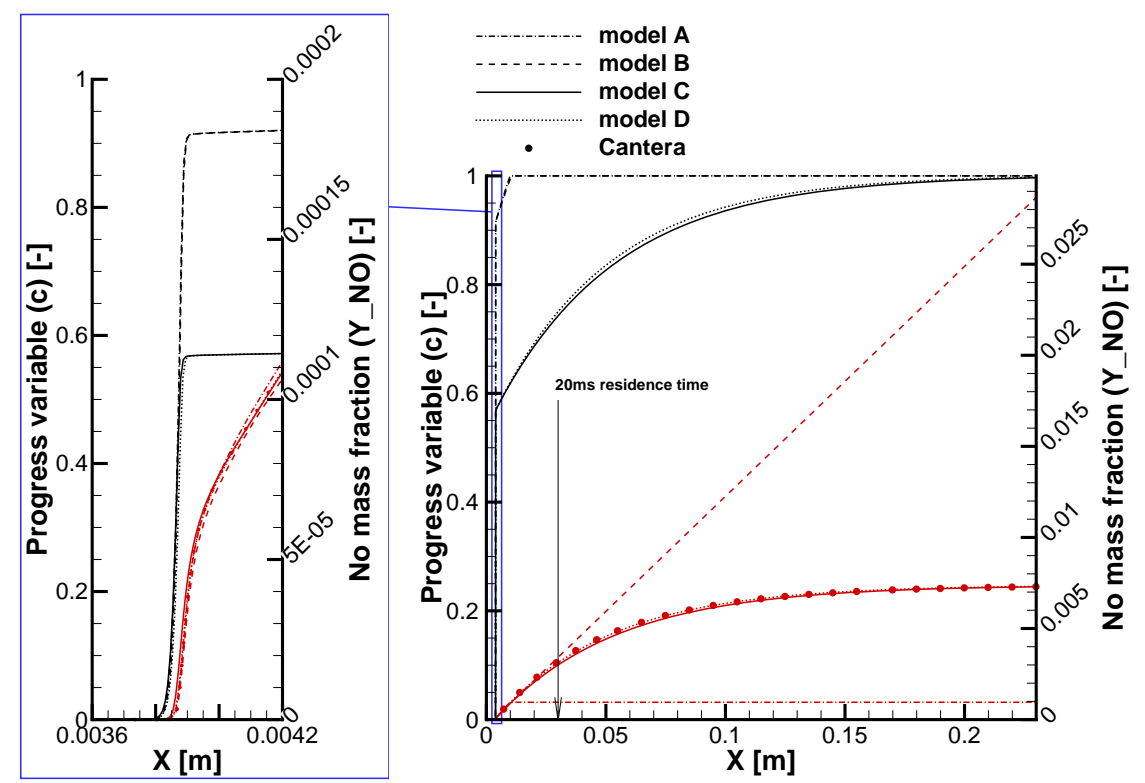

Note: Red lines stand for NO mass fraction while the black lines stand for the progress variable. 
Figure 4 presents the NO profiles obtained with the four approaches (models A to D) using the Cedre code, and the profile obtained directly with Cantera. As expected, with model $\mathrm{A}$, once the normalised progress variable reaches its maximum value of 1 , the constant net production term gives a linear evolution of the NO mass fraction leading to an important overestimation of this quantity. The abscissa where the residence time in the 1D flame reaches $20 \mathrm{~ms}$ is indicated in the figure. At this abscissa the overestimation of $Y_{N O}$ by the model $\mathrm{A}$ is limited. But, if we refer to the previous $0 \mathrm{D}$ computations, we can see that it was also the case in the PFR for the same FAR of 0.7 . Nevertheless, for higher FAR, the OD PFR calculations show a clear overestimation of $Y_{N O}$ in the same residence time. Since the evolution of NO whith model A in the current 1D flame with FAR of 0.7 is similar to its evolution in the $0 \mathrm{D}$ computation at same FAR, it is clear that the model A would largely overestimate NO for higher FAR in the 1D flame too. Model B, shows the same saturation effect as in our previous $0 D$ simulations once the progress variable reaches its maximum value of 1 . Models $C$ and $D$, with the extended manifold give the correct evolution of NO mass fraction. It is worth noting that model $\mathrm{B}$ and $\mathrm{D}$ are based on the same formulation of the NO source term and that the only difference is the use of the extended-manifold in model D. This points out that it is important to generate the manifold with its extended part.

\section{NO prediction in TAPS type burner simulation}

In order to illustrate the potential improvement in NO prediction resulting from the extension of the flamelet manifold, different NO models are tested on a semi-industrial configuration including a TAPS burner. The TAPS combustor is an injection concept tested on the Onera M1 facility, in the framework of the European project Towards Lean Combustion (2005). In this injection system, a premixed zone with a moderate fuel air ratio (FAR) is created by two annular swirlers. Air coming from these swirlers is mixed with the fuel that is injected through multiple small holes distributed on the injector circumference. In this lean zone, the mixture burns at relatively low temperatures, and this helps reduce $\mathrm{NO}_{\mathrm{x}}$ production. In order to stabilise the combustion for low power operation, a rich pilot zone is created on the axis of the burner by an atomiser injecting fuel droplets in a swirled air flow. Modelling such a configuration requires to tackle multiphysics problems since we are dealing with reactive turbulent two-phase flows. It is thus a very challenging target for application of a $\mathrm{NO}_{\mathrm{x}}$ model.

The conditions and the geometry have been simplified in order to reduce the calculations to simple 2D axisymmetric simulations. The computations are of RANS type in order to obtain short computational time, since we are only interested in the main tendencies given by the different models. We are focusing on long residence time effects, occurring downstream of the turbulent flame, where the mass fraction fluctuations have significantly decreased compared to their level in the main reaction zone. Therefore, we can assume that, in the post-flame zone, the turbulence-chemistry interaction is not modifying the tendencies concerning the NO formation, in such a way that we neglect the turbulence-chemistry interaction in the present calculations. Our methodology is however compatible with different methods used to take into account this interaction such as PCM-FPI (Fiorina et al., 2005), F-TACLES (Fiorina et al., 2010), TFLES/ATF (Kuenne et al., 2011). In the case of TFLES/ATF, the flame is artificially thickened independently of the type of table. In the filtered flame approach, the spatial 
filter used to filter the flamelet manifold can be used with the extended manifold as well. The PCM-FPI approach would require a bit more attention if the presumed PDF used for the progress variable is a $\beta$ function: it is more consistent to limit the convolution with a $\beta$-PDF to the 'initial flamelet' part of the manifold. Indeed, the $\beta$-PDF formalism is convenient for the flamelets structure, but not for PFR-like combustion as we have in the extended part of the manifold.

It is well known that radiative heat losses may have a strong impact on temperature in a combustion chamber, and thus, a strong impact on the NO production rates. Measurements achieved at Onera's M1 facility for different operating points of the TAPS burner during the TLC campaign (Towards Lean Combustion, 2005) show that soot concentration is very low in the combustion chamber. Since soot are usually the main source of radiative heat losses, the latter are expected to be low in this configuration. Moreover, 3D LES simulations of the TAPS burner configuration including radiative heat transfers have been done by Dorey (2012) for two operating points of the TAPS configuration. Both soot and gas radiation were included in the modelling, with an optically thick media assumption. Results of those simulations show that due to the low levels of soot concentration, the effect of radiative heat losses on the temperature is negligible. Hence, radiative heat fluxes are not considered in the current $2 \mathrm{D}$-axi simulations.

The global FAR in the present simulations is 0.5 . Liquid fuel droplets are injected in the swirler channels. A lagrangian solver is used to track these droplets. The gas-phase solver and the lagrangian solver are coupled through the evaporation term, the heat fluxes between droplets and gas-phase, and the drag force applied by the gas on the droplets. An unstructured mesh with 10000 triangular cells was generated. This relatively coarse grid has been designed to obtain tendencies of the models at low computational cost.

In the following, five NO models are compared (the labels of the models are kept same as in Table 2):

- NO interpolated directly in the not extended flamelet manifold (named hereafter model 0)

- $\quad \mathrm{NO}$ transported with the source term deduced from the not extended flamelet manifold as in Vreman et al. (2008) (i.e., model A)

- NO transported with the source term given by equations (8) and (9) and with the not extended flamelet manifold (i.e., model B)

- NO transported with the source term deduced from the extended manifold (i.e., model C)

- NO transported with the source term given by equations (8) and (9) and with the extended manifold (i.e., model D).

To compare quantitatively the different models, the average NO mass fraction in the outlet section of the TAPS burner configuration is computed for the five models as:

$$
\left\langle\overline{Y_{N O}}\right\rangle=\frac{\int_{0}^{R} r \bar{\rho} \overline{v_{x}} \overline{Y_{N O}} \cdot d r}{\int_{0}^{R} r \bar{\rho} \overline{v_{x}} \cdot d r}
$$


where $R$ is the radius of the combustor, $\bar{\rho}$ is the mean density and $\overline{v_{x}}$ is the mean axial velocity. Here, we assume that the scalar fluctuations are negligible in the outlet section. The same formulation applied to the local FAR gives an average FAR of 0.5 which is consistent with the global FAR. The average NO mass fractions obtained for the five models are summarised in Table 3. As an indication, calculated NO indices are also given in Table 3 . They are defined as

$$
I_{N O}[\mathrm{~g} / \mathrm{kg} \text { of fuel }]=1000 \times 2 \pi \frac{\int_{0}^{R} r \bar{\rho} \overline{Y_{N O}} \overline{v_{x}} \cdot d r}{\dot{m}_{f u e l}}
$$

where $\dot{m}_{f u e l}$ is the total mass flow rate of liquid fuel.

Table 3 NO indices, and NO average mass fraction in the outlet section of the TAPS burner configuration

\begin{tabular}{lcc}
\hline Approach for NO modelling & NO indice & Average NO mass fraction in outlet section \\
\hline Model 0 & 0.66 & $1.8 \times 10^{-05}$ \\
Model A & 25.67 & $6.9 \times 10^{-04}$ \\
Model B & 0.60 & $1.8 \times 10^{-05}$ \\
Model C & 5.65 & $1.5 \times 10^{-04}$ \\
Model D & 6.90 & $2.1 \times 10^{-04}$ \\
\hline
\end{tabular}

The NO mass fraction fields obtained with the five approaches for NO modelling are compared in Figures 5 and 6. The results of these simulations give a tendency coherent with the $0 \mathrm{D}$ and $1 \mathrm{D}$ simulations presented in the previous part of this paper. In Figure 5, the top field results from direct interpolation in the manifold (model 0 ) and corresponds to the field that would be obtained with a FGM/FPI model without any particular treatment for NO. In that case, NO is essentially present in the recirculation zone evidenced by the streamlines, and the average mass fraction reduces to $1.8 \times 10^{-05}$ in the outlet section. This value is not far from the maximum value of NO mass fraction obtained in the not extended manifold at the mixture fraction $\mathrm{Z}$ corresponding to the FAR of 0.5 , which is equal to $2.5 \times 10^{-5}$.

The middle field of Figure 5 is the solution obtained by transporting NO with the source term deduced from the not extended flamelet manifold (i.e., model A). As expected, the global level of NO is higher, with an average mass fraction of $6.9 \times 10^{-04}$ in the outlet section (38 times the level obtained with direct interpolation). This is induced by the possibility for the concentration of NO to linearly increase once the progress variable has reached its maximum value. Indeed, with the not extended manifold, the source term which is frozen and independent of the local conditions keeps non physical large values in the post-flame zone, leading to overestimated NO concentrations.

The lowest field of Figure 5 has been obtained with the extended table (i.e., model C). Compared to the top field results, NO is present in the convergent part of the domain, and in the outlet section. Nevertheless, the average mass fraction in the outlet section is lower $\left(1.5 \times 10^{-4}\right)$ than with the source term picked in the not extended manifold. The reason is that the source term taken in the extended manifold is linked on the local NO concentration through the progress variable, and thus decreases when the NO mass fraction increases. 
Figure 5 NO mass fraction field for three simulations of the TAPS configuration (see online version for colours)
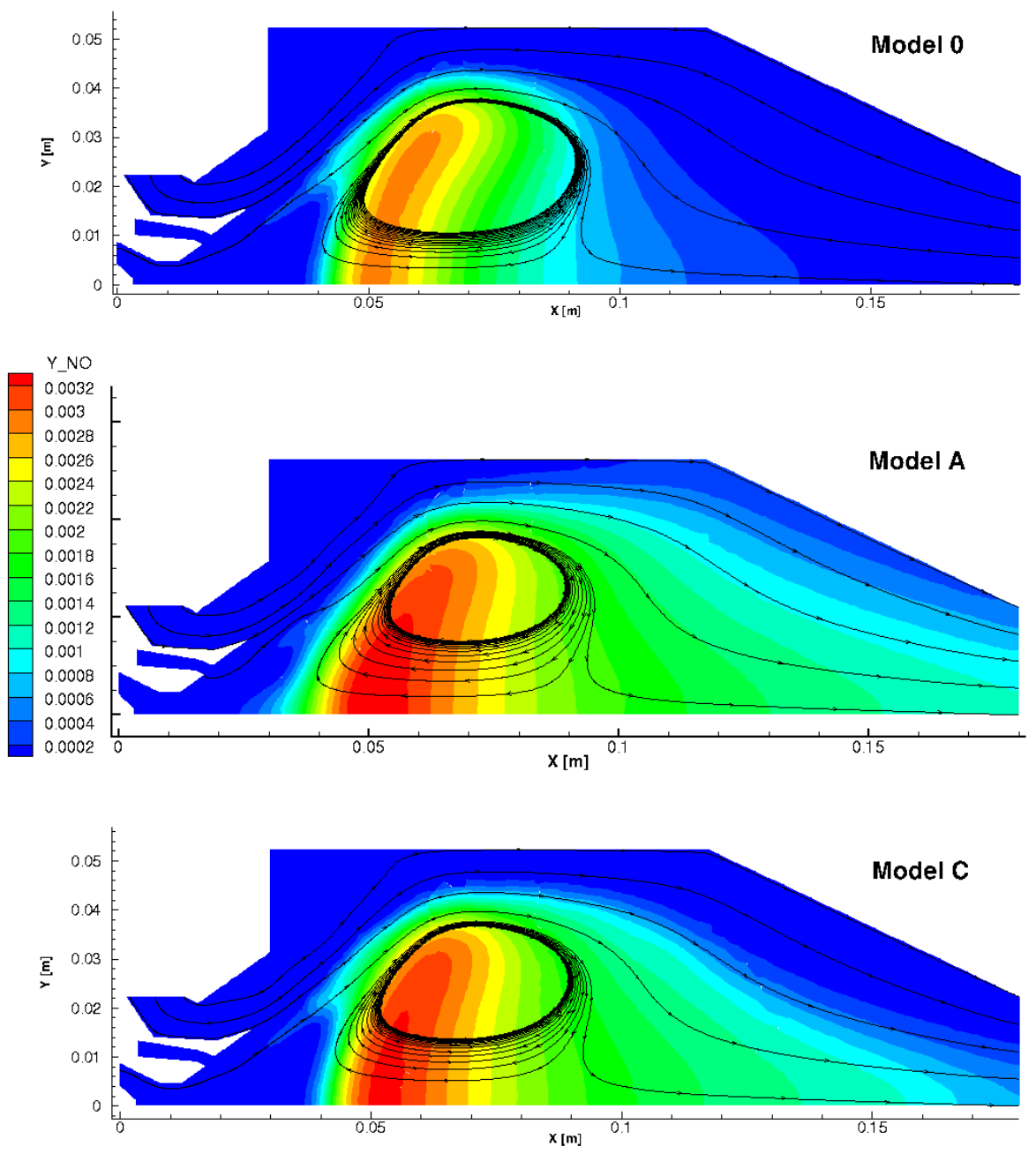

Notes: The colour scale is common to the three NO mass fraction fields. Top, tabulated chemistry ithout any method dedicated to NO, middle, model A, bottom, model $\mathrm{C}$.

In Figure 6, both fields were obtained with the source term given by equations (8) and (9). For the upper field, the not extended manifold was used (i.e., model B) and for the lower field, the extended manifold was used (i.e., model D). The predictions obtained with model B are very close to those obtained with model 0 , while model D gives results similar, though not identical, to those of model $\mathrm{C}$. This points out that in this configuration, the results are mainly influenced by the choice of the manifold. Hence, whatever the method used to calculate the source term, the PFR extension is essential to reproduce residence time effects in the industrial TAPS burner configuration. 
Figure 6 NO mass fraction field for two simulations of the TAPS configuration (see online version for colours)
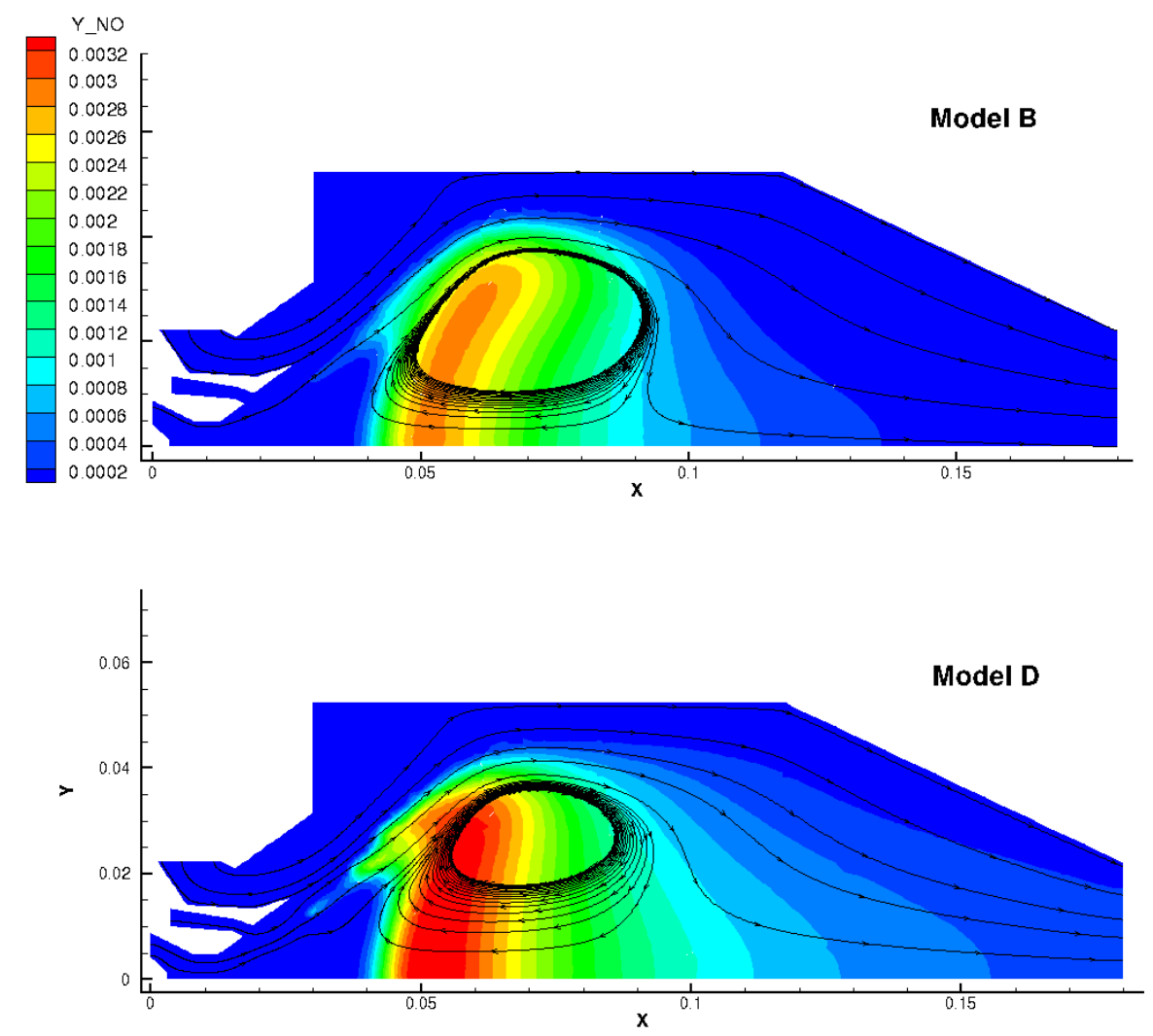

Notes: The colour scale is the same as Figure 5. On top, model B, below model D.

It is worth noting that the NO concentration obtained with the model $\mathrm{C}$ is very close to the NO concentration obtained by direct picking ${ }^{2}$ in the extended manifold, as shown in Figure 7. This indicates that the relaxation term used in model $\mathrm{C}$ [see equation (4)] to avoid deviations from tabulated trajectories has a correct effect on the solution.

A further analysis of the fields obtained with the model $\mathrm{C}$ has been carried out. The mean residence time $\tau$ and its standard deviation $\sigma_{\tau}$ were calculated from the residence time distribution (RTD) (Nauman, 2003). To evaluate the RTD, an unsteady simulation is done to record the flux for a passive scalar $(S)$ through the outlet section. Starting from the steady state solution, the scalar $S$ was injected at the three inlets, during a very short time (relatively to the expected residence time). Then, the unsteady RANS calculation was run during $0.1 \mathrm{~s}$, to completely evacuate the passive scalar from the domain. The evolution of the mass flux of this scalar $\Phi_{S}(t)$ versus time, in the outlet section, was recorded during the complete simulation. Hence, the mean residence time and its standard deviation were obtained from:

$$
\bar{\tau}=\frac{\int_{0}^{\infty} t \Phi_{S}(t) d t}{\int_{0}^{\infty} \Phi_{S}(t) d t}
$$


$\sigma_{\tau}=\left(\frac{\int_{0}^{\infty}(t-\bar{\tau})^{2} \Phi_{S}(t) d t}{\int_{0}^{\infty} \Phi_{S}(t) d t}\right)^{0.5}$

Figure 7 NO mass fraction field obtained with the extended manifold by solving an equation for NO (top) and by direct interpolation in the table (bottom) (see online version for colours)
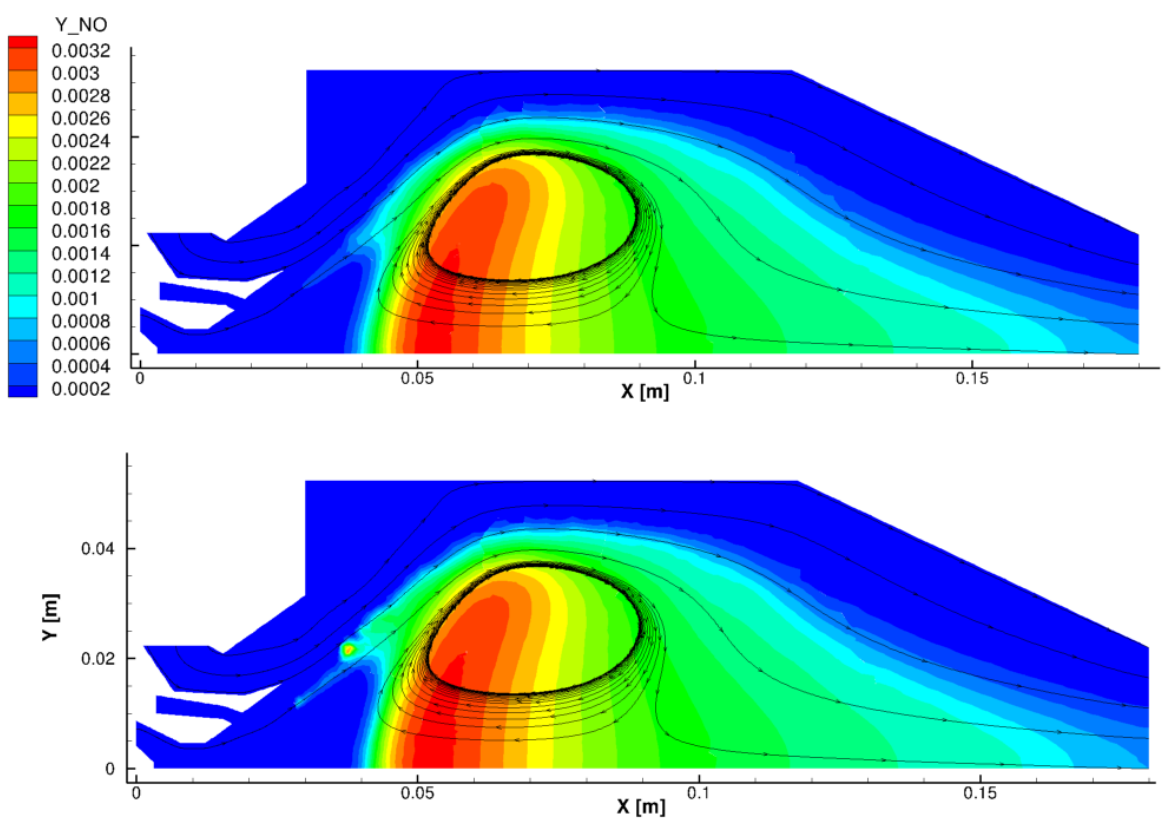

Note: The colour scale is common to the two NO mass fraction fields.

The mean residence time was found to be $4.0 \mathrm{~ms}$ with a standard deviation of $2.5 \mathrm{~ms}$. As mentioned earlier, the average NO mass fraction in the outlet section predicted by model $\mathrm{C}$ is $1.5 \times 10^{-04}$, with an average FAR of 0.5 . In the laminar unstretched premixed 1D flame used to generate the manifold, the NO mass fraction after $20 \mathrm{~ms}$ of residence time is only $3 \times 10^{-05}$ for the same FAR of 0.5 . To obtain a NO mass fraction of $1.5 \times 10^{-04}$ in the laminar 1D flame, we have to consider the abscissa where the residence time reaches $136 \mathrm{~ms}$, which is much larger than the average residence time in the outlet section of the TAPS burner. This comes from the fact that the predicted NO concentration in the outlet section is driven by the capacity of the model to take into account the FAR history in the fluid particles. Actually, NO is mainly produced in areas where the FAR is close to stoichiometry (see Figures 8 and 9). The NO mass fraction increases in these regions, in conjunction with the progress variable $Y_{c}^{\text {def } 3}$. Then, before reaching the outlet, the mixing reduces the FAR and the NO mass fraction, but this mass fraction remains much higher than the value that would be obtained at the outlet FAR with the same residence time. This explains why we obtain levels equivalent to those corresponding to residence time larger than $100 \mathrm{~ms}$ at a constant FAR of 0.5 , while the mean residence time is only $4.0 \mathrm{~ms}$. This mechanism of dilution near the outlet also 
explains why there is a saturation effect with model 0 and model $\mathrm{B}$, which both use the not extended manifold. Indeed, in those calculations, when the mixing reduces the FAR before reaching the outlet, it also decreases dramatically the value of NO mass fraction picked in the not extended manifold (as mentioned previously, the maximum NO mass fraction is equal to $2.5 \times 10^{-5}$ for the FAR of 0.5 in this manifold). Thus, the NO mass fraction is bounded by the tabulated value obtained at the maximum value of the progress variable even with the model $B$ based on the picking of the reaction rate but leading to a very slow NO evolution in the post-flame.

Figure 8 NO reaction rate field and FAR contour lines, obtained with model C (see online version for colours)

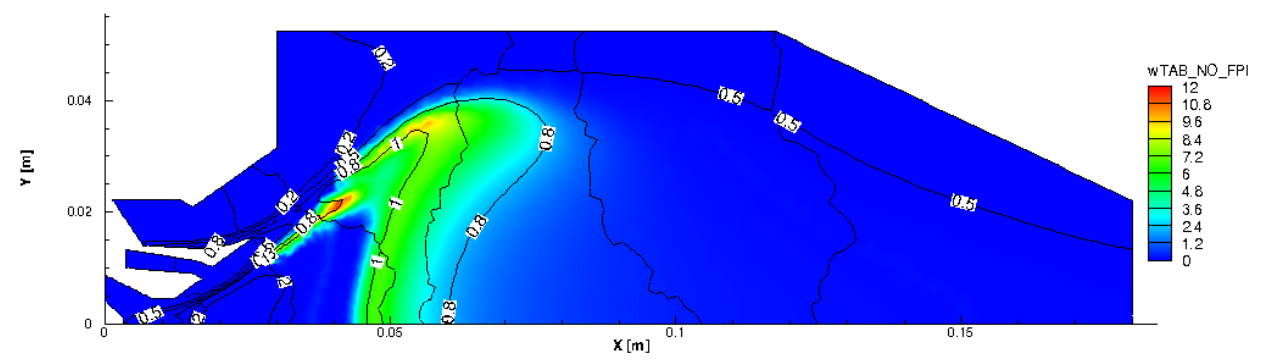

Figure 9 Cumulated NO source term $[\mathrm{kg} / \mathrm{s} / \mathrm{rad}]$ as function of FAR,weighted by the FAR section size (see online version for colours)

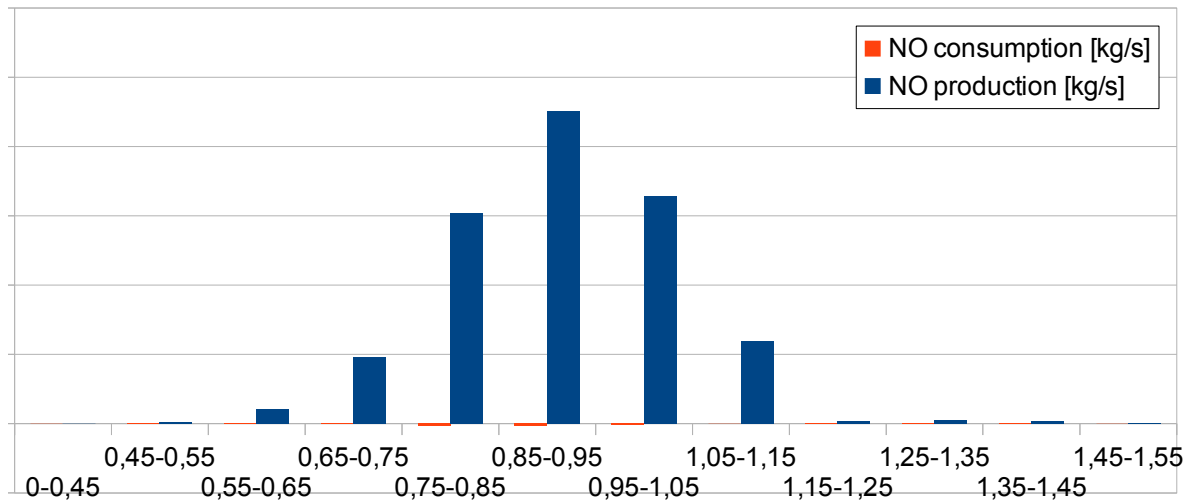

Figure 9 shows cumulated NO net production terms in FAR sections. Blue bars correspond to cells where the net production is positive (NO production) while orange bars correspond to cells where the net production is negative (NO consumption). Those cumulated terms are expressed as:

$$
\frac{\sum_{1}^{N}\left(\left(\overline{\dot{\omega}_{N O}} V\right) \mid \phi \in\left[\phi_{n} ; \phi_{n+1}[)\right.\right.}{\phi_{n+1}-\phi_{n}}
$$

with $N$ the number of cells of the mesh, $\phi_{n}$ and $\phi_{n+1}$ the lower and upper bounds of the FAR section, $V$ the volume per radian of the cell, and $\overline{\dot{\omega}}_{N O}$ the NO consumption or 
production rate in the cell. NO is mainly produced in areas where the FAR is close to 0.95 , and the weight of the area where the net NO source term is negative is negligible.

The validation of the method by comparison with the experiment is required. For that, it is necessary to realise 3D LES computations to correctly capture the mixing by the large scales of turbulence. The turbulence-chemistry interaction must be taken into account, to accurately predict the reaction rates where the flame is submitted to high turbulence levels. This will have an influence on NO predictions since prompt NO is produced in those areas. Although the complexity of these rigorous validations have been left aside in the present paper, the 2D axi-symmetric simulations of the TAPS burner configuration yield important conclusions. They clearly show that, to calculate such type of combustion chambers, it is necessary to turn to chemistry tables taking into account large residence times and the PFR extension is particularly adapted to such configurations.

\section{Conclusions}

The FPI/FGM tabulated chemistry model gives rise to difficulties when some species have an evolution time greater than the main species. Some methods are proposed in the literature to improve the modelling of the NO formation. Nevertheless they have been shown unable to solve this problem in the case of flows having quite large residence time, or when the FAR stratification of the flow leads to NO concentrations equivalent to those obtained with a large residence time at constant FAR. A new method has been proposed to extend the validity of the manifold used in a tabulated chemistry model, in order to take into account species having a long evolution time such as NO. This method can be implemented very easily with a negligible additional CPU cost and gives encouraging results on the simulation of a TAPS burner. The simplicity and the low CPU cost of the proposed method give us the possibility to introduce an additional entry in the chemical table to take into account thermal losses if necessary, as it has already been done with the not extended manifold. In addition, it is compatible with existing turbulence/chemistry interaction closures. A further work of validation by comparison with experimental results has still to be done, and requires more expensive $3 \mathrm{D}$ calculations.

\section{References}

Baba, Y. and Kurose, R. (2008) 'Analysis and flamelet modelling for spray combustion', Journal of Fluid Mechanics, Vol. 612, October, pp.45-79."

Dorey, L-H. (2012) Modelling of Combustion, Soot Formation and Radiative Transfer Coupled Phenomena in Gas Turbine Combustion Chambers, PhD thesis, Ecole Centrale Paris, Châtenay-Malabry, France [online] http://tel.archives-ouvertes.fr/tel-00731144.

Fiorina, B., Gicquel, O., Vervisch, L., Carpentier, S. and Darabiha, N. (2005) 'Premixed turbulent combustion modeling using tabulated detailed chemistry and PDF', Proceedings of the Combustion Institute, Vol. 30, No. 1, pp.867-874.

Fiorina, B., Vicquelin, R., Auzillon, P., Darabiha, N., Gicquel, O. and Veynante, D. (2010) 'A filtered tabulated chemistry model for LES of premixed combustion', Combustion and Flame, Vol. 157, No. 3, pp.465-475. 
Galpin, J. (2007) LES Modelling of Combustion Taking into Account Detailed Kinetic Effects and with the Perspective of Piston Engine Applications, $\mathrm{PhD}$ thesis, INSA de Rouen, Saint-Etienne du Rouvray, Fance [online] http://www.theses.fr/2007ISAM0018.

Gicquel, O., Darabiha, N. and D. Théveninn (2000) 'Laminar premixed hydrogen/air counterflow flame simulations using flame prolongation of ILDM with differential diffusion', Proceedings of the Combustion Institute, Vol. 28, No. 2, pp.1901-1908.

Godel, G., Domingo, P. and Vervisch, L. (2009) 'Tabulation of NOx chemistry for large-eddy simulation of non-premixed turbulent flames', Proceedings of the Combustion Institute, Vol. 32, No. 1, pp.1555-1561.

Goodwin, D., Malaya, N., Moffat, H. and Speth, R. (2013) 'Cantera: an object-oriented software toolkit for chemical kinetics, thermodynamics, and transport processes', v2.1a [online] https://code.google.com/p/cantera/ (accessed 2014-04-01).

Ihme, M. and Pitsch, H. (2008) 'Modeling of radiation and nitric oxide formation in turbulent nonpremixed flames using a flamelet/progress variable formulation', Physics of Fluids, Vol. 20, No. 5, pp.055110-055110-20.

Ketelheun, A., Olbricht, C., Hahn, F. and Janicka, J. (2011) 'NO prediction in turbulent flames using LES/FGM with additional transport equations', Proceedings of the Combustion Institute, Vol. 33, No. 2, pp.2975-2982.

Kuenne, G., Ketelheun, A. and Janicka, J. (2011) 'LES modeling of premixed combustion using a thickened flame approach coupled with FGM tabulated chemistry', Combustion and Flame, Vol. 158, No. 9, pp.1750-1767.

Luche, J. (2003) Elaboration of Reduced Kinetic Models of Combustion - Application to a Kerosene Mechanism, $\mathrm{PhD}$ thesis, University of Orléans, France [online] http://tel.archives-ouvertes.fr/tel-00636023.

Nauman, E.B. (2003) 'Residence time distributions', in E.L. Paul, V.A. Atiemo-Obeng and S.M. Kresta (Eds.): Handbook of Industrial Mixing, pp.1-17, John Wiley \& Sons, Inc.

Savre, J., Bertier, N., D'Angelo, Y. and Gaffié, D. (2008) 'A chemical time scale approach for FPI modeling', Comptes Rendus Mécanique, Vol. 336, Nos. 11-12, pp.807-812.

Towards Lean Combustion (2005) [online] http://ec.europa.eu/research/transport/projects/items/tlc_en.htm (accessed 2014-04-01).

Van Oijen, J., Lammers, F. and de Goey, L. (2001) 'Modeling of complex premixed burner systems by using flamelet-generated manifolds', Combustion and Flame, Vol. 127, No. 3, pp.2124-2134.

Van Oijen, J.A. and De Goey, L.P.H. (2009) 'Predicting no formation with flamelet generated manifolds', in Proceedings of the European Combustion Meeting.

Vreman, A., Albrecht, B., van Oijen,J., de Goey, L. and Bastiaans, R. (2008) 'Premixed and nonpremixed generated manifolds in large-eddy simulation of sandia flame $\mathrm{d}$ and $\mathrm{f}$ ', Combustion and Flame, Vol. 153, No. 3, pp.394-416.

\section{Notes}

1 Actually, enthalpy losses can appear locally. This can then be taken into account by adding the enthalpy coordinate to the extended flamelet manifold, but this is beyond the scope of this paper.

2 The calculations presented here were done with the indirect approach as presented in the first section. Nevertheless, the tabulated mass fractions are stored in the manifold, and thus the field given by direct interpolation in the manifold can be calculated. 


\section{Nomenclature}

\begin{tabular}{ll}
\hline$\dot{\omega}_{i}$ & Reaction rate \\
$\mathcal{M}_{i}$ & Molecular weight of species $i$ \\
$\phi$ & Fuel-air ratio \\
$\rho$ & Density \\
$\tau$ & Characteristic time \\
$c$ & Dimensionless progress variable \\
$T$ & Temperature \\
$t$ & Time coordinate \\
$V$ & Volume \\
$x, y, z$ & Spatial coordinates \\
$Y_{c}$ & Progress variable \\
$Y_{i}$ & Mass fraction of the species $i$ \\
$Z$ & Mixture fraction \\
F-TACLES & Filtered tabulated chemistry for large eddy simulation \\
FAR & (turbulent combustion model) \\
FGM & Fuel air ratio \\
FPI & Flamelet generated manifold (combustion model) \\
LES & Flame prolongation of ILDM (combustion model) \\
NO & Large eddy simulation \\
NO & Nitric oxide \\
PDF & Nitrogen oxides \\
PFR & Probability density function \\
RANS & Plug flow reactor \\
RTD & Reynolds average Navier-Stokes \\
TAPS & Residence time distribution \\
TFLES & Twin annular premixing swirler \\
TLC & Thickened flame for large eddy simulation \\
\hline & (chemistry-turbulence interaction model) \\
& Towards lean combustion (european project) \\
&
\end{tabular}

